\title{
Relationship of possible biomarkers with malignancy of thymic tumors: a meta- analysis
}

Huilan Zeng ${ }^{1+}$, Weilin Yang ${ }^{1}$, Bo Xu' ${ }^{1}$, Jianyong Zou ${ }^{2}$, Chunhua Su ${ }^{2}$ Beilong Zhong ${ }^{3}$, Haoshuai Zhu ${ }^{2}$ and Zhenguang Chen ${ }^{1,2,3^{*}+}$

\begin{abstract}
Background: Role of biomarkers for promotion of tumor proliferation (BPTPS) and for promotion of apoptosis (BPAs) in thymic malignant tumors is still unclear. The purpose of this study was to evaluate the relationship between BPTPs and/or BPAs and malignancy of thymic malignant tumors.

Methods: Studies on thymic malignant tumors and biomarkers were searched in PubMed, ISI Web of Knowledge, and Embase databases, and all statistical analyses were conducted using Review Manager.

Results: Twelve articles related to biomarkers and thymic malignant tumors were selected and analyzed. A relationship between BPAs and Masaoka stage was demonstrated for four markers, namely Bax, p73, Casp-9 and BCl-2, included 138 stage I/II patients and 74 stage III/IV patients, and BPAs were significantly correlated with high Masaoka staging $(P=0.03)$. We further found a relationship between BPAs and degree of malignancy for four markers, namely Bax, p73, Casp-9 and Bcl-2, included 176 thymoma patients and 36 thymic carcinoma patients, and BPAs were significantly correlated with thymic carcinoma $(P=0.010)$. In addition, a relationship between BPTP and Masaoka staging was demonstrated for seven markers, namely Podoplanin, Glut-1, Muc-1, Egfr, Igf1r, c-Jun, and nRas, included 373 patients with stage I/II and 212 patients with stage III/IV, and BPTPs were significantly correlated with high Masaoka staging $(P<0.001)$. We also found a relationship between BPTPs and degree of malignancy for ten markers, namely Mesothelin, c-Kit (CD117), Egfr, Lat-1, Muc-1,Ema, Glut-1, Igf1r, c-Jun, and n-Ras, included 748 thymoma patients and 280 thymic carcinoma patients, and BPTPs were significantly correlated with thymic carcinoma $(P<0.001)$.

Conclusion: These findings show that high levels of BPTPs or BPAs are more closely related to thymic carcinoma and Masaoka stage III/IV, suggesting that BPTPs and BPAs may play an important role in the occurrence and development of thymic malignant tumors.
\end{abstract}

Keywords: Biomarkers, Proliferation, Apoptosis, Thymic malignant tumors, Malignancy

\footnotetext{
* Correspondence: chenzhenguang@yahoo.com

${ }^{\dagger}$ Huilan Zeng and Zhenguang Chen contributed equally to this work.

'Department of Thoracic Surgery and Department of Cardiothoracic Surgery

of East Division, the First Affiliated Hospital of Sun Yat-Sen University,

Guangzhou, No. 58, Zhongshan Road II, Guangzhou, Guangdong 510080, P.

R. China

${ }^{2}$ Department of Thoracic Surgery, the First Affiliated Hospital of Sun Yat-Sen

University, Guangzhou, Guangdong 510080, P. R. China

Full list of author information is available at the end of the article
}

(C) The Author(s). 2020 Open Access This article is licensed under a Creative Commons Attribution 4.0 International License, which permits use, sharing, adaptation, distribution and reproduction in any medium or format, as long as you give appropriate credit to the original author(s) and the source, provide a link to the Creative Commons licence, and indicate if changes were made. The images or other third party material in this article are included in the article's Creative Commons. licence, unless indicated otherwise in a credit line to the material. If material is not included in the article's Creative Commons licence and your intended use is not permitted by statutory regulation or exceeds the permitted use, you will need to obtain permission directly from the copyright holder. To view a copy of this licence, visit http://creativecommons.org/licenses/by/4.0/ The Creative Commons Public Domain Dedication waiver (http://creativecommons.org/publicdomain/zero/1.0/) applies to the data made available in this article, unless otherwise stated in a credit line to the data. 


\section{Background}

Thymic malignant tumors, including malignant thymoma and thymic carcinoma, are a group of rare diseases with unknown etiology that are often diagnosed based on histological criteria. Both of these malignancies, especially thymic carcinoma, show cytologic atypia, invasive margins, and loss of an organotypic appearance [1]. It is generally considered that the biological characteristics of malignant thymoma and thymic carcinoma are related to their staging and pathological malignancy, and that the prognosis of surgical resection for malignant thymoma and thymic carcinoma is worse than that for thymoma in the early phase [1-7]. Unfortunately, because of a lack of uniform measurement standards, determining the prognosis of malignant thymoma and thymic carcinoma based on an analysis of histological type is complicated. One of the main features of the present system for classifying thymomas, as exemplified by the World Health Organization (WHO) classification system, is the proportion of lymphocytes in the thymus tumor. According to this system, WHO type A and type B1 are believed to be less invasive, whereas type B2 and type B3 are considered to be more invasive [8-11].

Recent studies have explored the relationship between the expression of several biomarkers and the prognosis or diagnosis of malignancy in thymic malignant tumors. These markers include EGFR (epidermal growth factor receptor), GLUT1 (glucose transporter 1), EMA (epithelial membrane antigen), IGF-1R (insulin-like growth factor I receptor), BAX (BCL2 associated X), p73, BCL2 (B cell leukemia/lymphoma 2), PD-L1 (programmed death ligand 1), FOXP3 (forkhead box P3) and TdT (terminal deoxynucleotidyl transferase), among others [12-21]. In addition, increased expression of tumor-associated genes, such as FPGS (folylpolyglutamate synthase)/GGH (gamma-glutamyl hydrolase) and VEGF (vascular endothelial growth factor), were found to be related to the degree of malignancy in thymic carcinoma and B3 thymoma [8]. Notably, C-kit expression, which is detectable in approximately $70-86 \%$ of patients with thymic carcinoma, is only found in $0-5 \%$ of thymic adenomas $[10,22]$. The epigenetics of thymoma genes has also been investigated, including histone modification, chromatin recombination, and gene methylation $[11,22]$. However, although such studies have identified a number of biomarkers related to thymic malignant tumors, they have often reported quite different or even completely opposite results and have sampled limited population sizes [16, 22-28]. Because of these limiting factors, there is no definitive conclusion as to which markers are capable of reflecting the degree of malignancy or Masaoka stage of thymomas.

On the other hand, some biomarkers, such as EGFR, GLUT-1 and IGF-1R, have similar characteristics that impact the proliferative potential and invasive ability of tumors. Mutation of the apoptosis-related proteins, BCL-2, p53 or BAX, can lead to the occurrence and development of thymic malignant tumors. Expression of FOXP3 and TdT in cancer cells suggests that $\mathrm{T}$ cells may play a part in tumor immune escape. Given their similar characteristics, Podoplanin, Muc-1, Glut-1, Egfr, Igf1r, c-Jun, n-Ras, Mesothelin, c-Kit (cd117), Lat1, and Ema, have been classified as biomarkers for promotion of tumor proliferation (BPTPs); Bax, p73, Casp-9 and Bcl-2 have been classified as biomarkers for promotion of tumor apoptosis (BPAs); and FOXP3 and TdT have been classified as $\mathrm{T}$ cell markers. Given the relative lack of literature on the relationship of $\mathrm{T}$ cell markers, B cell markers and mitogenic markers with thymic malignant tumors, studies on the association of BPAs or BPTPs with thymic malignant tumors are particularly noteworthy.

Strong expression of BPAs is associated with advanced thymoma and thymic carcinoma [13]. Studies have shown that thymic malignant tumors expressing BPTPs tend to be advanced and highly malignant $[12,14,17,18,23,29-$ 33]. However, this conclusion is tempered by the small sample sizes involved and discrepancies among reports. For example, the frequency of BPA or BPTP positivity in Masaoka stage III/IV was showed in higher level than that in stage I/II, but it was also found no statistically significant difference in BPA or BPTP positivity according to Masaoka stages [13] Similarly, the frequency of BPA or BPTP positivity in thymic carcinoma was showed higher level than that in thymoma, but other studies found no significant difference between these tumor types [12, 13, 34]. Along the same lines, it suggested that the rate of apoptosis in thymoma is higher than that in thymic carcinoma [22].

Given the high expression of numerous molecular markers in a variety of cancers and their association with poor prognosis, as well as controversies surrounding the significance of their expression in thymic epithelial

Table 1 Characteristics of the studies included in the meta-analysis: relationship between BPAs and Masaoka staging

\begin{tabular}{llllll}
\hline First author & Year & Country & Ethnicity & No. of patients & Median age (years) \\
\hline Kenzo Hiroshima [16] & 2002 & Japan & Japanese & 46 & 56.3 \\
Yuqing Ma [17] & 2012 & China & Chinese & 60 & 48.5 \\
\hline
\end{tabular}


Table 2 Characteristics of the studies included in the meta-analysis: relationship between BPAs and degree of malignancy

\begin{tabular}{llllll}
\hline First author & Year & Country & Ethnicity & No. of patients & Median age (years) \\
\hline Kenzo Hiroshima [16] & 2002 & Japan & Japanese & 46 & 56.3 \\
Yuqing Ma [17] & 2012 & China & Chinese & 60 & 48.5
\end{tabular}

tumors, we conducted this study to determine whether BPTPs and/or BPAs contribute to the staging and degree of malignancy of thymic malignant tumors.

\section{Methods}

\section{Search strategy}

A comprehensive literature review in PubMed, ISI Web of Knowledge, and Embase databases was conducted using the key words "markers", "thymoma", "thymic adenocarcinoma" and "thymic malignant tumor". Articles published as of December 30, 2018, were collected, including case-control and cohort studies on thymoma and tumor markers.

\section{Inclusion criteria}

The following criteria were used to select documents for further meta-analysis: (1) published in English, regardless of the publication time; (2) evaluated the relationship between BPTPs (e.g., EGFR, GLUT-1, IGF-1R) or BPAs (e.g., Bcl-2, p73, Bax) and cancer stage or degree of malignancy; (3) confirmed cancer patients by pathology; (4) including detailed cancer/Masaoka staging data; and (5) divided thymic malignant tumor patients into at least two groups, namely, thymic adenocarcinoma versus thymic carcinoma or Masaoka stage I/II versus stage III/IV.

\section{Data extraction}

Two researchers independently reviewed each article under the guidance of an instructor from the same center. Details of publication characteristics for each qualified publication, including the first author's name, year of publication, patient's country of origin and race, total number of patients, cancer type, and median/average age and disease stage of the study population, were collected. Positive or high levels of markers and Masaoka staging or degree of malignancy were the focus of attention.

\section{Statistical analysis}

Four different relationships were analyzed: (1) the relationship between BPAs and Masaoka stage; (2) the relationship between BPAs and thymoma malignancy; (3) the relationship between BPTPs and Masaoka stage; and (4) the relationship between BPTPs and thymoma malignancy. Correlations between marker positivity/high expression and degree of malignancy and stage of thymic malignant tumors were determined by measuring odds ratios (ORs) and relevant 95\% confidence intervals (CIs). Statistical heterogeneity between studies was evaluated using Cochran's heterogeneity statistics Q and I2, which describe the variation caused by heterogeneity rather than random error, as follows: $\mathrm{I} 2=0-25 \%$, no heterogeneity; I2 = 25-50\%, moderate heterogeneity; I $2=50$ $75 \%$, large heterogeneity; and I $2=75-100 \%$, extreme heterogeneity. In the initial analysis, a fixed effects model was applied; a confirmed random effects model was used in cases where there was significant heterogeneity. A funnel chart was used to evaluate publication deviation. All statistical analyses were conducted using Review Manager Version 5.0 (RevMan Cochrane Collaboration, Oxford, UK). All $P$-values in meta-analysis were bilateral, and $P$-values less than 0.05 were considered significant.

\section{Results}

\section{Research characteristics}

Our initial search strategy identified 1774 potentially related studies. 260 repeated articles were deleted and the remaining 1514 articles were included in the initial study. After reading the title and abstract, we selected 112 articles that met our search criteria for further detailed evaluation. After careful screening, 97 studies were excluded because the markers in these studies were not related to tumor proliferation or apoptosis. Of the remaining 15 studies, three did not have sufficient data. In the end, 12 studies of markers and degree of malignancy or tumor stage were considered qualified for final analysis. The characteristics of the included studies are

Table 3 Characteristics of the studies included in the meta-analysis: relationship between BPTPs and Masaoka staging

\begin{tabular}{|c|c|c|c|c|c|}
\hline First author & Year & Country & Ethnicity & No. of patients & Median age (years) \\
\hline T. Mimae [12] & 2012 & Japan & Japanese & 140 & 54 \\
\hline Hisashi Tateyama [30] & 2011 & Japan & Japanese & 99 & 54 \\
\hline Yuqing Ma [17] & 2012 & China & Chinese & 60 & 48.5 \\
\hline Jun Du [13] & 2016 & China & Chinese & 43 & 51 \\
\hline
\end{tabular}


Table 4 Characteristics of the studies included in the meta-analysis: relationship between BPTPs and degree of malignancy

\begin{tabular}{|c|c|c|c|c|c|}
\hline First author & Year & Country & Ethnicity & No. of patients & Median age (years) \\
\hline Lchiro Fukai [14] & 1992 & Japan & Japanese & 95 & NR \\
\hline T. Mimae [12] & 2012 & Japan & Japanese & 140 & 54 \\
\hline Kiyotaka Yoh [16] & 2008 & Japan & Japanese & 38 & 61 \\
\hline Kyoichi Kaira [32] & 2011 & Japan & Japanese & 55 & 61 \\
\hline Kyoichi Kaira [33] & 2009 & Japan & Japanese & 45 & 55 \\
\hline Chinchen Pan [35] & 2004 & China & Chinese & 132 & NR \\
\hline Anish Thomas [36] & 2016 & America & American & 71 & 51 \\
\hline Yuqing Ma [17] & 2012 & China & Chinese & 60 & 48.5 \\
\hline Daisuke Nonaka [23] & 2007 & America & American & 75 & 59 \\
\hline Jun Du [13] & 2016 & China & Chinese & 43 & 51 \\
\hline
\end{tabular}

listed in Tables 1, 2, 3, 4. PRISMA flow-diagram was shown in Fig. 1.

\section{Publication bias}

Funnel charts of the four groups are shown in the Figure. No obvious asymmetry was detectable in any of the four groups, demonstrating the absence of publication bias.

\section{Relationship between BPAs and Masaoka staging}

Four markers, namely Bax, p73, Casp-9 and Bcl-2, from two articles that included 138 patients in phase I/II and 74 patients in phase III/IV were selected. Combining the

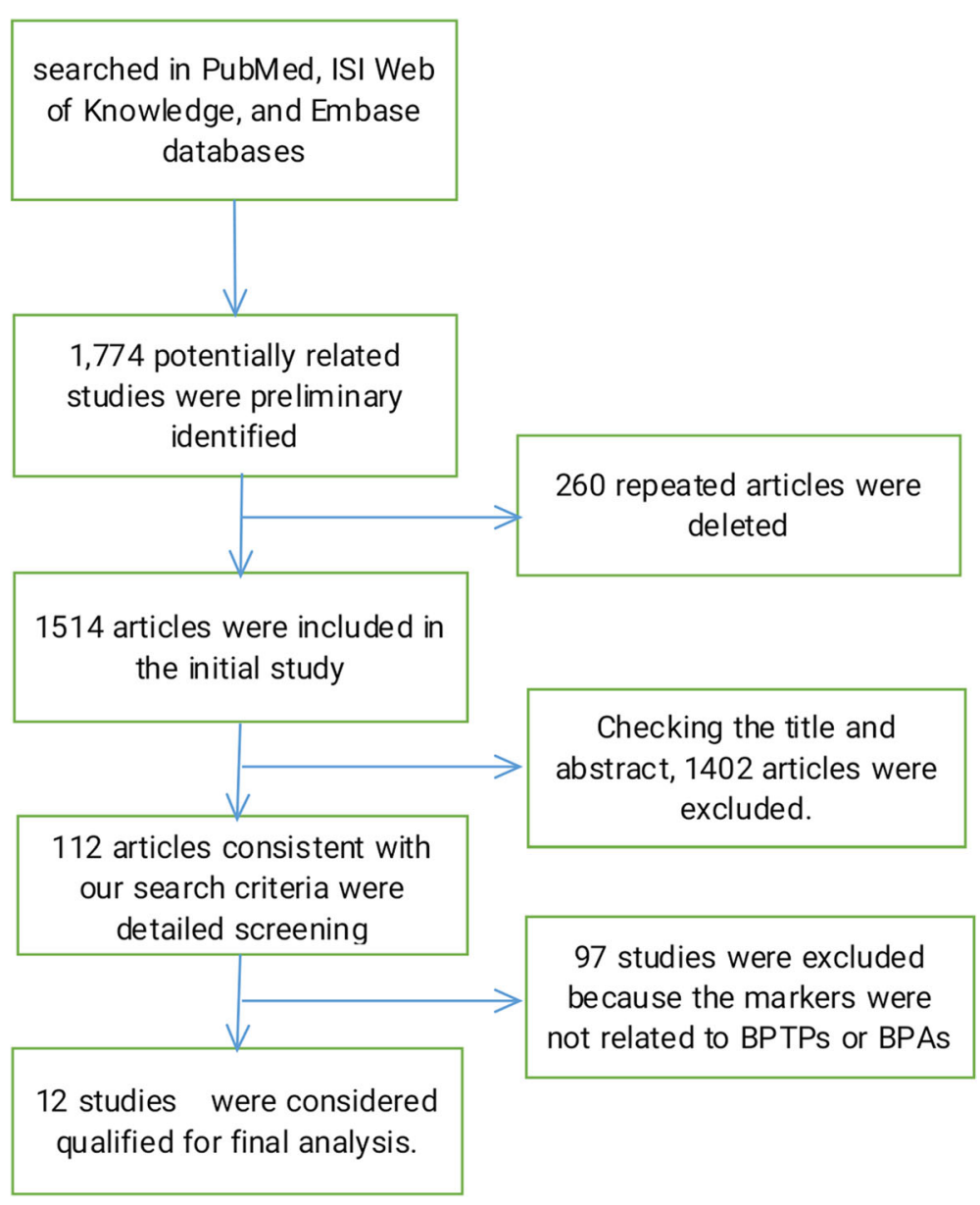

Fig. 1 PRISMA flow-diagram 
A

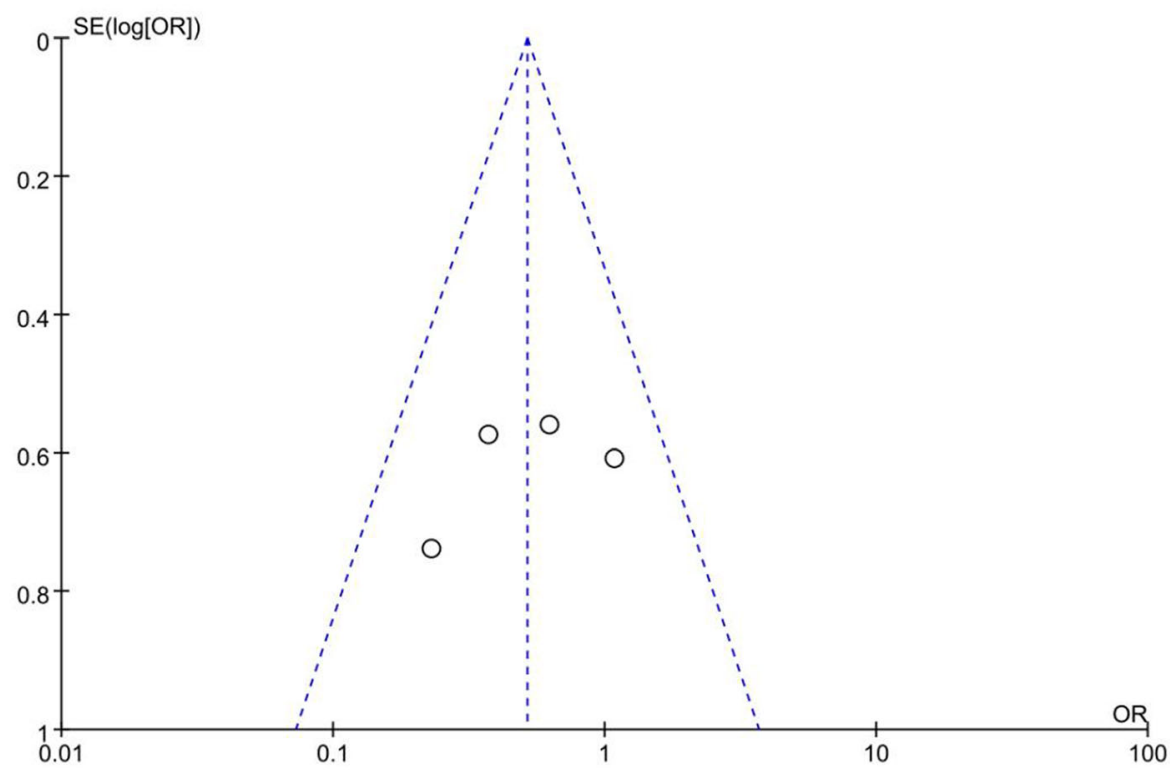

B

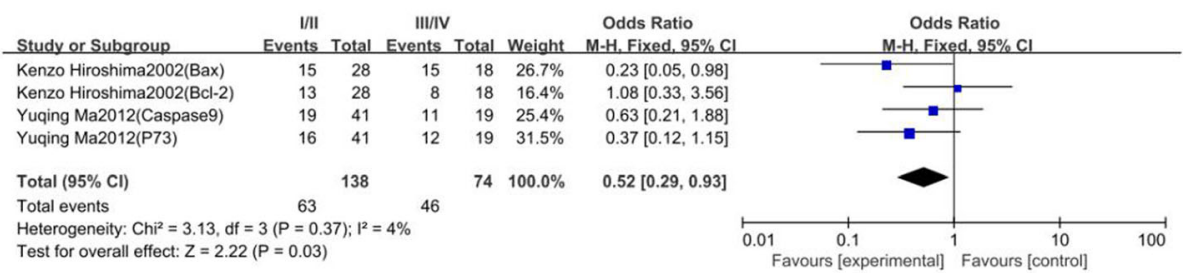

Fig. 2 Relationship between BPAs and Masaoka staging. a, Funnel plot. b, Forest plot

results from these two eligible studies in a meta-analysis revealed evidence of a correlation between positive/ highly expressed pro-apoptotic tumor markers and thymoma stage III/IV. As shown in Fig. 2b, significant major effects were observed between positive/highly expressed BPAs and Masaoka stage III/IV (I/II vs. III/IV: OR 0.52, 95\% CI 0.29-0.93; $P=0.03$ ).

\section{Relationship between BPAs and degree of malignancy}

Four markers, namely Bax, p73, Casp-9 and Bcl-2, from two articles that included 176 cases of thymoma and 36 cases of thymic carcinoma were selected. As shown in Fig. 3b, significant major effects were observed between positive/highly expressed BPAs and thymic carcinoma (thymoma vs. thymic carcinoma: OR $0.36,95 \%$ CI $0.17-$ $0.79 ; P=0.01)$.

\section{Relationship between BPTP and Masaoka staging}

Seven markers, namely Podoplanin, Glut-1, Muc-1, Egfr, Igf1r, c-Jun, and n-Ras, from five articles that included 373 patients in phase I/II and 212 patients in phase III/
IV were selected. As shown in Fig. 4b, significant major effects were observed between positive/highly expressed BPTPs and Masaoka stage III/IV (I/II vs. III/IV: OR 0.34, 95\% CI 0.23-0.50; $P<0.00001$ ).

Relationship between BPTPs and degree of malignancy Ten markers, namely Podoplanin, Glut-1, Muc-1, Egfr, Igf1r, c-Jun, and n-Ras, from ten articles that included 748 cases of thymoma and 280 cases of thymic carcinoma were selected. As shown in Fig. 5b, significant major effects were observed between positive/highly expressed BPTPs and thymic carcinoma (thymoma vs. thymic carcinoma: OR $0.07,95 \%$ CI $0.04-0.10$; $P<0.00001)$.

\section{Heterogeneity test}

Using Q statistics and applying a random-effects model, we observed statistically significant heterogeneity between the following tests: BPAs and thymoma versus thymic carcinoma $(P=0.09, \mathrm{I} 2=54 \%)$; $\mathrm{BPTPs}$ and phase $\mathrm{I} / \mathrm{II}$ versus phase III/IV $(P<0.00001, \mathrm{I} 2=82 \%)$; and BPTPs and 
A

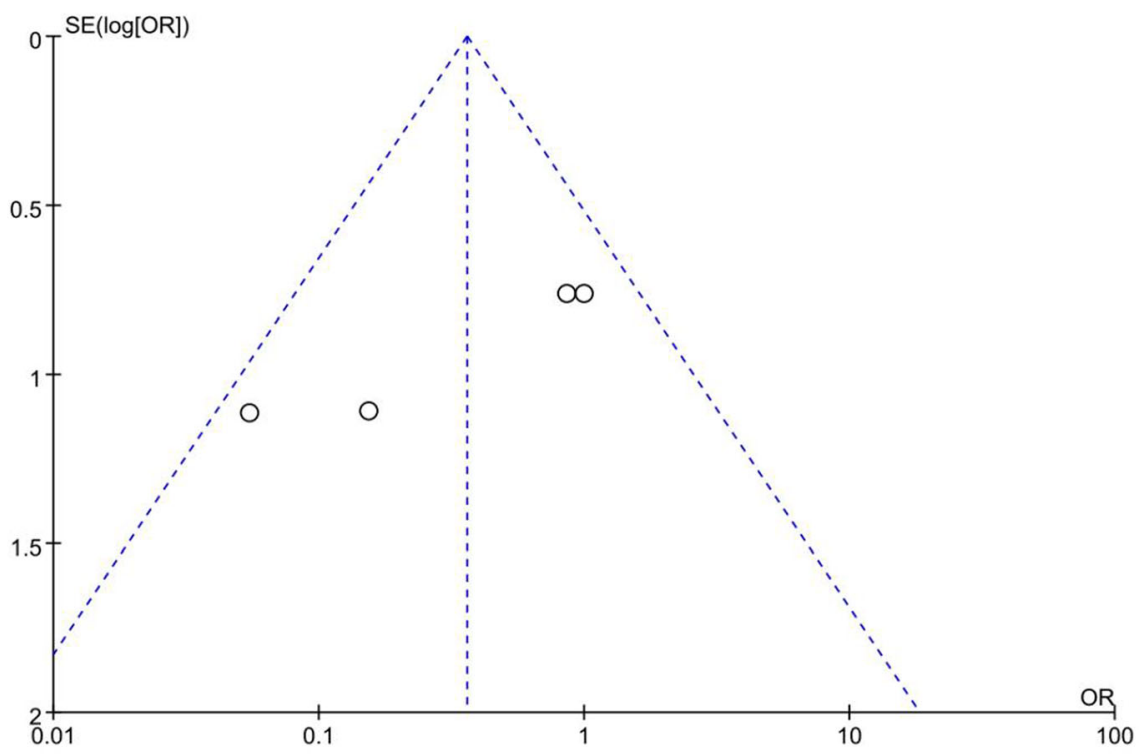

B

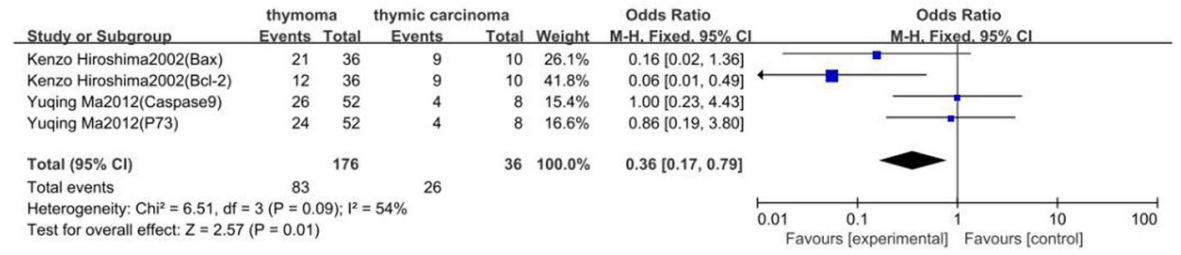

Fig. 3 Relationship between BPAs and degree of malignancy. a, Funnel plot. b, Forest plot

thymoma versus thymic carcinoma $(P<0.00001, \mathrm{I} 2=$ $85 \%)$. We found no obvious heterogeneity between BPAs and Masaoka stage $(P=0.75, \mathrm{I} 2=0 \%)$; therefore, a fixed effect model was used for this analysis.

\section{Discussion}

An excess of bax protein promotes apoptosis. Proteins of the B-cell lymphoma-2 (BCL-2) family control the intrinsic apoptosis pathway. The pro-apoptotic BCL-2 proteins BAX and BAK can commit a cell to its programmed death by permeabilizing the outer mitochondrial membrane $(\mathrm{OMM})$ and subsequent initiation of the caspase cascade [16, 37].

Apoptosis or programmed cell death is a fundamental cellular process that is paramount for cellular regeneration and tissue homeostasis in multicellular organisms. Unlike other cell death pathways, apoptosis efficiently dismantles the cell without adverse effects on neighboring cells or its environment. Its faithful execution is essential in avoiding a number of catastrophic disease states and is also critical in organismal development, so apoptosis is very tightly regulated. Caspases, aspartate-directed, cysteine proteases play prominent roles in apoptotic pathways. Initiator caspases (caspase-2, -8 and -
9) function upstream of the apoptotic pathways while executioners (caspase-3, -6 and -7) mediate downstream reactions [35]. As part of the p53 family, with different kinds of promoter transcription and alternative splicing, p73 can produce $>10$ different subtypes, collectively called DNp73 or $\triangle \mathrm{TAp} 73$, that play an important role in the expression of human tumors. The DNp73 is different from p53 in function due to the significant difference in their structures, while the $\triangle$ TAp73 has similar functions as p53 in inhibiting tumor and promoting apoptosis [36].

Given their similar characteristics, Bax, p73, Casp-9 and Bcl-2 have been classified as biomarkers for promotion of tumor apoptosis (BPAs) in this study.

In head and neck squamous cell carcinoma, overexpression of podoplanin is associated with lymph node metastasis and poor clinical outcome. MUC1 oncoprotein is aberrantly expressed at high levels in most human neoplasms, and MUC1 plays important roles in development and progression of malignant tumors. Several studies have identified GLUT1 as a prognostic and diagnostic marker and it has been found to be associated with tumor progression and poor overall survival in various malignant tumors $[18,29]$. 
A

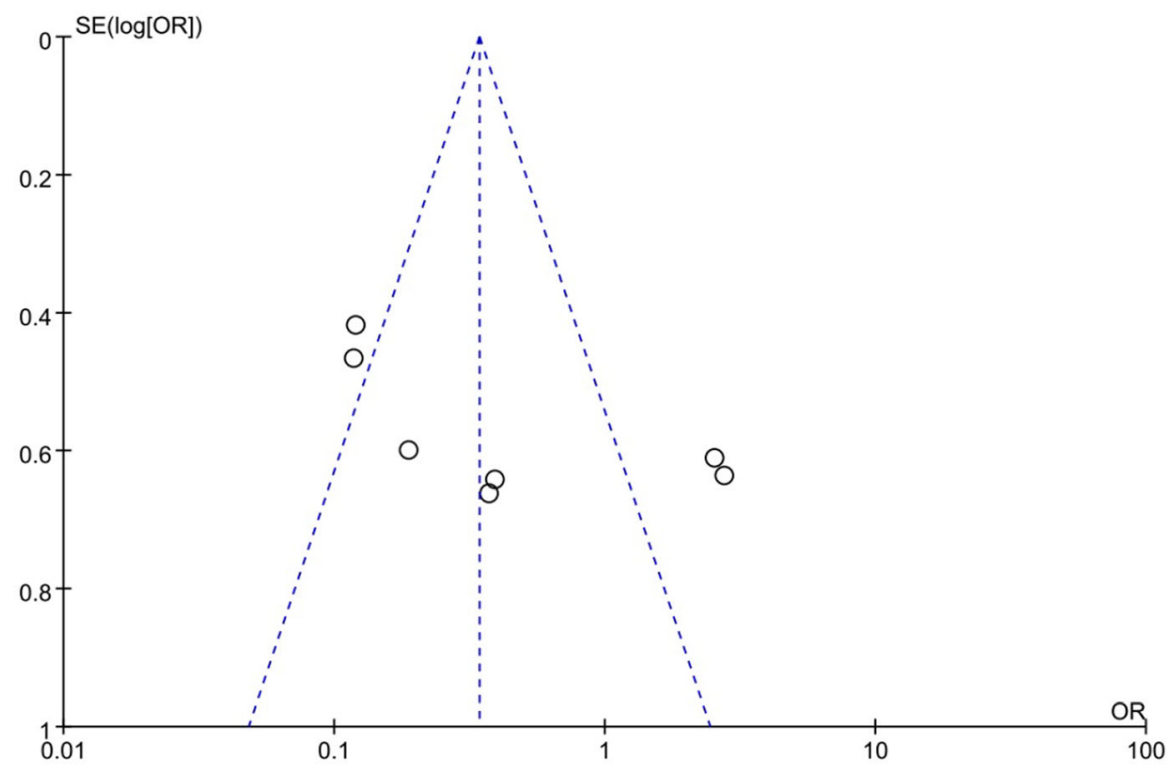

B

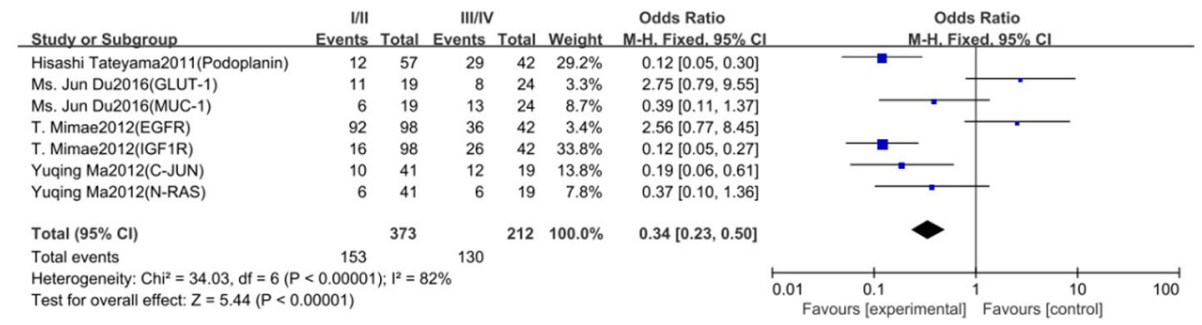

Fig. 4 Relationship between BPTPs and Masaoka staging. a, Funnel plot. b, Forest plot

Insulin-like growth factor-1 receptor (IGF-1R), epidermal growth factor receptor (EGFR), human epidermal growth factor receptor-type 2 (HER2), and c-Met are members of the receptor tyrosine kinases (RTKs). Receptor tyrosine kinases (RTKs) have been shown to have critical roles in the proliferation, migration, and survival of many types of malignant neoplastic cells. C-Jun can directly act as an oncogene by helping in the proliferation and consequent invasion and metastasis of the cells. Zheng ZY found that wild-type N-RAS is overexpressed in BLBCs. Repressing N-RAS inhibits transformation and tumor growth, whereas overexpression enhances these processes even in preinvasive BLBC cells. Mesothelin is a cell-surface antigen implicated in tumor invasion, which is highly expressed in mesothelioma, lung, pancreas, breast, ovarian, and other cancers [12, 38, 39].

CD117 is involved in the development of several malignant tumor types including gastrointestinal stromal cell tumors, small-cell lung, ovarian and breast cancer. Immunohistochemical staining has revealed that CD117 protein is overexpressed in primary malignant tumors, including operable esophageal squamous cell carcinoma and vulvar melanoma, and may be a valuable prognostic marker in esophageal squamous cell carcinoma. L-type amino acid transporter 1(Lat-1) is one of the amino acid transporters, which are necessary for tumor growth and proliferation, and is highly expressed in many cancer cells. In addition, LAT1 was highly expressed in patients with pancreatic cancer, and its expression yielded a significant association with cell proliferation, angiogenesis, and disease stage. Sloane and Ormerod reported that EMA staining of various tumors is related to the degree of tumor differentiation. The increase of EMA expression after malignant neoplastic transformation is suggested to be related to poor intercellular contact, which may help to sustain the unrestricted growth characteristic of neoplasms [14, 40, 41].

Given their similar characteristics, Bax, p73, Casp-9 and $\mathrm{Bcl}-2$ have been classified as biomarkers for promotion of tumor apoptosis (BPAs); Podoplanin, Muc-1, Glut-1, Egfr,Igf1r, c-Jun, n-Ras, Mesothelin, c-Kit 


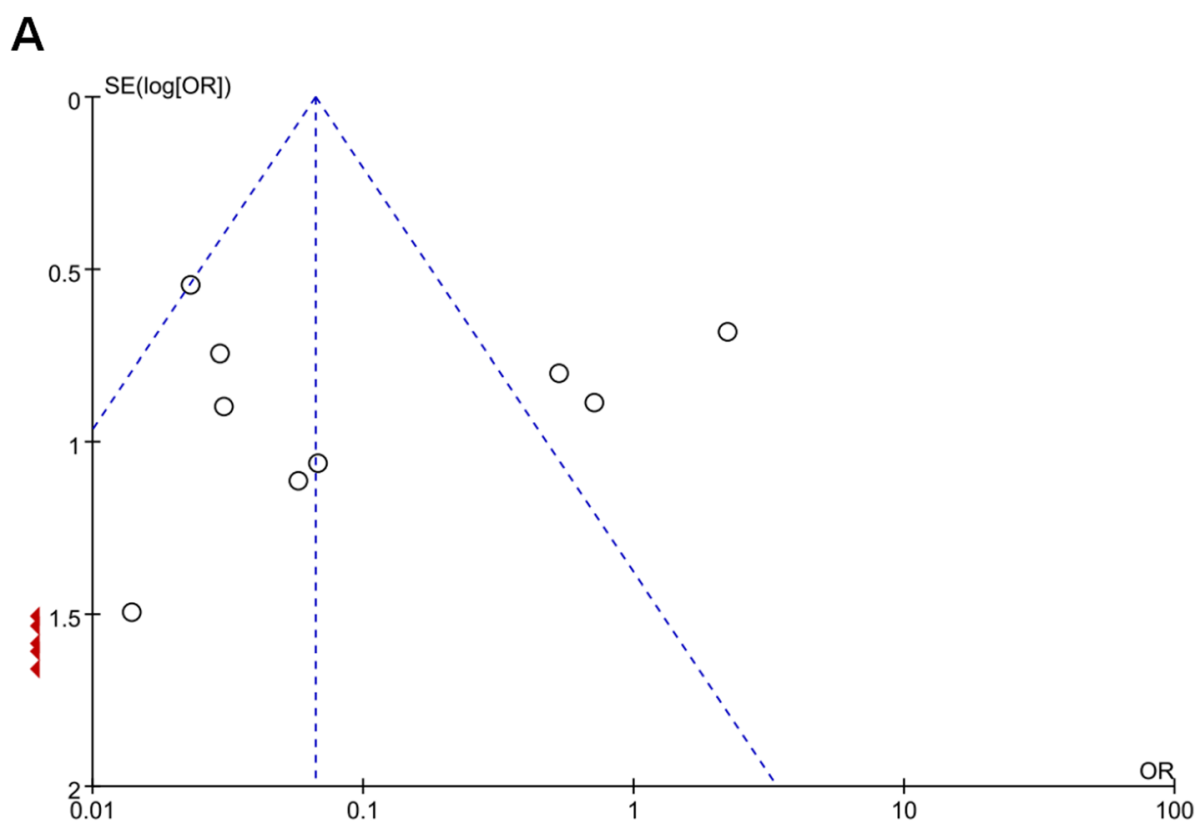

B

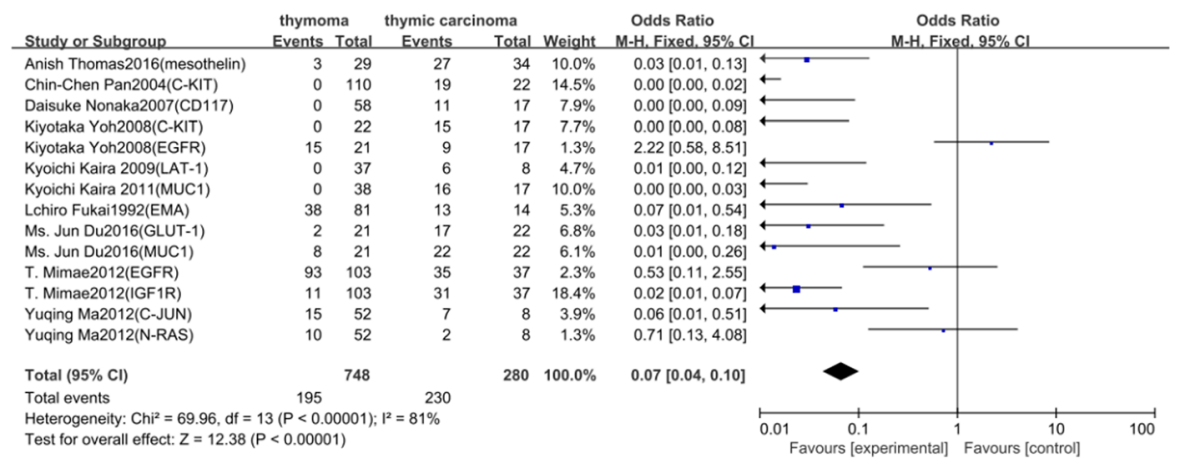

Fig. 5 Relationship between BPTPs and degree of malignancy. a, Funnel plot. b, Forest plot

(cd117), Lat1, and Ema, have been classified as biomarkers for promotion of tumor proliferation (BPTPs).

BPAs and/or BPTPs are often used to predict the occurrence and development of malignancy and monitor effects of therapy on tumors. Such biomarkers have been widely used in the diagnosis and treatment of malignant tumors. Examples include treatment of EGFRmutation-positive lung cancer with mutant-EGFR-targeted inhibitors, or elevation of AFP as an indicator of liver cancer. However, the mechanisms underlying the involvement of BPAs and BPTPs in the development of thymic malignant tumors, especially their relationship to clinical stage or pathological malignancy, have remained unclear. In the present study, we systematically identified and evaluated existing data, and analyzed the relationships between the rate of tumor marker positivity, reflecting tumor proliferation and apoptosis, and thymoma versus cancer and phase I/II versus phase III /IV. These analyses revealed evidence of a significant association of BPAs or BPTPs with thymic adenocarcinoma or stage III/IV thymic malignant tumors.

Our demonstration of a significant relationship between positivity/high expression of BPAs or BPTPs and thymic adenocarcinoma or stage III/IV thymic malignant tumors suggests that gene products that promote tumor proliferation or apoptosis may play an important role in the occurrence and development of thymic malignant tumors. However, further investigation of thymic malignant tumors is needed to confirm our results. We will continue to do further research. Analyse patient's sample with Histo-pathological technique and real time PCR using BPTPS and BPA markers, Using thymoma patient specimens for total gene detection. If the results of both of them also indicate that the staging and malignancy of thymoma are related to BPTPs or BPAs, our conclusion will be more effectively verified. 


\section{Conclusions}

Collectively, our findings provide evidence of a correlation between expression of BPAs or BPTPs and Masaoka staging or pathological malignancy of thymoma. Therefore, high levels or positive expression of BPAs or BPTPs may be strongly linked to high Masaoka stages of thymoma or more prominent pathological malignancy.

\section{Abbreviations}

BPTPs: Biomarkers for promotion of tumor proliferation; BPAs: Biomarkers for promotion of apoptosis; GLUT-1: Glucose transporter 1; EGFR: Epidermal growth factor receptor; IGF1R: Insulin-like growth factor-1 receptor; LAT1: Ltype amino acid transporter 1; EMA: Epithelial membrane antigen

\section{Acknowledgments}

We declare no conflicts of interest in connection with the current study.

\section{Authors' contributions}

Conceived and designed the experiments: $\mathrm{HZ} 1$ and ZC. Performed the experiments and acquired data: $\mathrm{HZ} 1, W Y, B X, J Z, C S, B Z$, and HZ2. Analyzed the data: $\mathrm{HZ}$ and $\mathrm{ZC}$. Wrote the paper: $\mathrm{HZ1}$ and $\mathrm{ZC}$. All authors read and approved the final manuscript.

\section{Funding}

This study was not supported by any grants.

\section{Availability of data and materials}

Data sharing not applicable to this article as no datasets were generated or analyzed during the current study.

\section{Ethics approval and consent to participate}

Not applicable.

\section{Consent for publication}

Not applicable.

\section{Competing interests}

The authors declare that they have no competing interests.

\section{Author details}

${ }^{1}$ Department of Thoracic Surgery and Department of Cardiothoracic Surgery of East Division, the First Affiliated Hospital of Sun Yat-Sen University, Guangzhou, No. 58, Zhongshan Road II, Guangzhou, Guangdong 510080, P. R. China. ${ }^{2}$ Department of Thoracic Surgery, the First Affiliated Hospital of Sun Yat-Sen University, Guangzhou, Guangdong 510080, P. R. China. ${ }^{3}$ Department of Thoracic Surgery, the Fifth Affiliated Hospital of Sun Yat-Sen University, Zhuhai, Guangdong 519000, P. R. China.

\section{Received: 27 June 2019 Accepted: 24 August 2020}

Published online: 29 September 2020

\section{References}

1. Weissferdt A, Moran CA. Thymic carcinoma, part 1: a clinicopathologic and immunohistochemical study of 65 cases. Am J Clin Pathol. 2012;138:103-14.

2. Burt BM, Yao X, Shrager J, Antonicelli A, Padda S, Reiss J, Wakelee H, Stacey S, Huang J, Scott W. Determinants of complete resection of Thymoma by minimally invasive and open Thymectomy: analysis of an international registry. J Thorac Oncol. 2017;12(1):129-36.

3. Hosaka Y, Tsuchida M, Toyabe S, Umezu H, Eimoto T, Hayashi J. Masaoka stage and histologic grade predict prognosis in patients with thymic carcinoma. Ann Thorac Surg. 2010;89(3):912-7.

4. Xue L, Wang L, Dong J, Yuan Y, Fan H, Zhang Y, Wang Q, Ding J. Risk factors of myasthenic crisis after thymectomy for thymoma patients with myasthenia gravis. Eur J Cardiothorac Surg. 2017;52(4):692-7.

5. Aydiner A, Toker A, Sen F, Bicakci E, Saglam EK, Erus S, Eralp Y, Tas F, Oral EN, Topuz E, Dilege $S$. Association of clinical and pathological variables with survival in thymoma. Med Oncol. 2012;29(3):2221-8.
6. Bae MK, Byun CS, Lee CY, Lee JG, Park IK, Kim DJ, Yang WI, Chung KY. Clinical outcomes and prognosis of recurrent thymoma management. J Thorac Oncol. 2012;7(8):1304-14.

7. Zhu L, Zhang J, Marx A, Weiss C, Fang WT. Clinicopathological analysis of 241 thymic epithelial tumors-experience in the Shanghai chest hospital from 1997-2004. J Thorac Dis. 2016:8(4):718-26.

8. Li Q, Su YL, Shen SWXW. A novel prognostic signature of seven genes for the prediction in patients with thymoma. J Cancer Res Clin Oncol. 2019; 145(1):109-16.

9. Cappuzzo F, Hirsch FR, Rossi E, Bartolini S, Ceresoli GL, Bemis L, Haney J, Witta S, Danenberg K, Domenichini I, Ludovini V, Magrini E, Gregorc V, Doglioni C, Sidoni A, Tonato M, Franklin WA, Crino L, Bunn PA Jr, Varella-Garcia M. Epidermal growth factor receptor gene and protein and gefitinib sensitivity in non-small-cell lung cancer. J Natl Cancer Inst. 2005;97(9):643-55.

10. Henley JD, Cummings OW, Loehrer PJ Sr. Tyrosine kinase receptor expression in thymomas. J Cancer Res Clin Oncol. 2004;130(4):222-4.

11. Gümüstas S, Akça A, Inan N, Akgül AG, Liman ST. Characterization of malignant thrombus in an invasive thymoma with intravascular growth. J Radiol Case Rep. 2013:7(2):17-23.

12. Mimae T, Tsuta K, Kondo T, Nitta H, Grogan TM, Okada M, Asamura H, Tsuda H. Protein expression and gene copy number changes of receptor tyrosine kinase in thymomas and thymic carcinomas. Ann Oncol. 2012;23(12):3129-37.

13. Du MJ, Shen Q, Yin H, Rao Q, Zhou MX. Diagnostic roles of MUC1 and GLUT1 in differentiating thymic carcinoma from type B3 thymoma. Pathol Res Pract. 2016;212(11):1048-51.

14. Fukai I, Masaoka A, Hashimoto T, Yamakawa Y, Mizuno T, Tanamura O. The distribution of epithelial membrane antigen in thymic epithelial neoplasms. Cancer. 1992;70(8):2077-81.

15. Omatsu M, Kunimura T, Mikogami T, Hamatani S, Shiokawa A, Masunaga A, Kitami A, Suzuki T, Kadokura M, Morohoshi T. Immunohistochemical analysis of thymic carcinoma focusing on the possibility of molecular targeted and hormonal therapies. Gen Thorac Cardiovasc Surg. 2012;60(12):803-10.

16. Hiroshima K, lyoda A, Toyozaki T, Supriatna Y, Shibuya K, Shimamura F, Haga Y, Yoshida S, Fujisawa T, Ohwada H. Proliferative activity and apoptosis in thymic epithelial neoplasms. Mod Pathol. 2002;15(12):1326-32.

17. Ma Y, Li Q, Cui W, Miao N, Liu X, Zhang W, Zhang C, Wang J. Expression of c-Jun, p73, Casp9, and N-ras in thymic epithelial tumors: relationship with the current WHO classification systems. Diagn Pathol. 2012;7:120.

18. Kaira K, Murakami H, Serizawa M, Koh Y, Abe M, Ohde Y, Takahashi T, Kondo H, Nakajima T, Yamamoto N. MUC1 expression in thymic epithelial tumors: MUC1 may be useful marker as differential diagnosis between type B3 thymoma and thymic carcinoma. Virchows Arch. 2011;458(5):615-20.

19. Chen Y, Zhang Y, Chai X, Gao J, Chen G, Zhang W, Zhang Y. Correlation between the expression of PD-L1 and Clinicopathological features in patients with Thymic epithelial tumors. Biomed Res Int. 2018;2018:5830547.

20. Nishi T, Yokoyama S, Takamori S, Matsuo T, Murakami D, Akagi Y, Ohshima $K$. Thymoma in patient with myasthenia gravis has significantly fewer Forkhead box P3 positive lymphocytes than that without one. Kurume Med J. 2015;61(3-4):65-71.

21. Su XY, Wang WY, Li JN, Liao DY, Wu WL, Li GD. Immunohistochemical differentiation between type B3 thymomas and thymic squamous cell carcinomas. Int J Clin Exp Pathol. 2015;8(5):5354-62.

22. Badve S, Goswami C, Gökmen-Polar Y, Nelson RP Jr, Henley J, Miller N, Zaheer NA, Sledge GW Jr, Li L, Kesler KA, Loehrer PJ Sr. Molecular analysis of thymoma. PLoS One. 2012;7(8):e42669.

23. Pan CC, Chen PC, Chou TY, Chiang H. Expression of calretinin and other mesothelioma-related markers in thymic carcinoma and thymoma. Hum Pathol. 2003;34(11):1155-62.

24. Khoury T, Chandrasekhar R, Wilding G, Tan D, Cheney RT. Tumour eosinophilia combined with an immunohistochemistry panel is useful in the differentiation of type B3 thymoma from thymic carcinoma. Int J Exp Pathol. 2011,92(2):87-96.

25. Pan CC, Ho DM, Chen WY, Huang CW, Chiang H. Ki67 labelling index correlates with stage and histology but not significantly with prognosis in thymoma. Histopathology. 1998;33(5):453-8.

26. Roden AC, Yi ES, Jenkins SM, Donovan JL, Cassivi SD, Garces YI, Marks RS, Aubry MC. Diagnostic significance of cell kinetic parameters in World Health Organization type a and B3 thymomas and thymic carcinomas. Hum Pathol. 2015:46(1):17-25.

27. Nonaka D, Henley JD, Chiriboga L, Yee H. Diagnostic utility of thymic epithelial markers CD205 (DEC205) and Foxn1 in thymic epithelial neoplasms. Am J Surg Pathol. 2007;31(7):1038-44. 
28. Schirosi L, Nannini N, Nicoli D, Cavazza A, Valli R, Buti S, Garagnani L, Sartori G, Calabrese F, Marchetti A, Buttitta F, Felicioni L, Migaldi M, Rea F, Di Chiara F, Mengoli MC, Rossi G. Activating c-KIT mutations in a subset of thymic carcinoma and response to different c-KIT inhibitors. Ann Oncol. 2012;23(9): 2409-14.

29. Tateyama H, Sugiura H, Yamatani C, Yano M. Expression of podoplanin in thymoma: its correlation with tumor invasion, nodal metastasis, and poor clinical outcome. Hum Pathol. 2011;42(4):533-40.

30. Kaira K, Oriuchi N, Imai H, Shimizu K, Yanagitani N, Sunaga N, Hisada T, Ishizuka T, Kanai Y, Endou H, Nakajima T, Mori M. L-type amino acid transporter 1 (LAT1) is frequently expressed in thymic carcinomas but is absent in thymomas. J Surg Oncol. 2009;99(7):433-8.

31. Yoh K, Nishiwaki Y, Ishii G, Goto K, Kubota K, Ohmatsu H, Niho S, Nagai K, Saijo N. Mutational status of EGFR and KIT in thymoma and thymic carcinoma. Lung Cancer. 2008:62(3):316-20.

32. Pan CC, Chen PC, Chiang H. KIT (CD117) is frequently overexpressed in thymic carcinomas but is absent in thymomas. J Pathol. 2004;202(3):375-81.

33. Thomas A, Chen Y, Berman A, Schrump DS, Giaccone G, Pastan I, Venzon DJ, Liewehr DJ, Steinberg SM, Miettinen M, Hassan R, Rajan A. Expression of mesothelin in thymic carcinoma and its potential therapeutic significance. Lung Cancer. 2016;101:104-10.

34. Hosomi Y, Watanabe K, Yamada Y, Horio H, Maeda Y, Okamura T, Hishima T. Clinicopathological analysis of thymic malignancies with a consistent retrospective database in a single institution: from Tokyo metropolitan Cancer center. BMC Cancer. 2014;14:349.

35. Huber KL, Serrano BP, Hardy JA. Caspase-9 CARD : core domain interactions require a properly formed active site. Biochem J. 2018;475(6):1177-96.

36. Song DJ, Yue LF, Zhang D, Yang HY, Fan YX, Yue M, Pei H, Wang JX. Relationship between mRNA expression and promoter methylation status of p73 gene in peripheral blood among children with Wilms' tumor. Zhongguo Dang Dai Er Ke Za Zhi. 2013;15:638-43.

37. Edlich F. BCL-2 proteins and apoptosis: recent insights and unknowns. Biochem Biophys Res Commun. 2018;500(1):26-34.

38. Priyadarshini R, Hussain M, Attri P, Kaur E, Tripathi V, Priya S, Dhapola P, Saha D, Madhavan V, Chowdhury S, Sengupta S. BLM potentiates c-Jun degradation and alters its function as an oncogenic transcription factor. Cell Rep. 2018;24(4):947-61.

39. Zheng ZY, Tian L, Bu W, Fan C, Gao X, Wang H, Liao YH, Li Y, Lewis MT, Edwards D, Zwaka TP, Hilsenbeck SG, Medina D, Perou CM, Creighton CJ, Zhang XH, Chang EC. Wild-type N-Ras, overexpressed in basal-like breast Cancer, promotes tumor formation by inducing IL-8 secretion via JAK2 activation. Cell Rep. 2015;12(3):511-24.

40. Luo Y, Huang W, Zhang H, Liu G. Prognostic significance of CD117 expression and TP53 missense mutations in triple-negative breast cancer. Oncol Lett. 2018;15(5):6161-70

41. Altan B, Kaira K, Watanabe A, Kubo N, Bao P, Dolgormaa G, Bilguun EO, Araki K, Kanai Y, Yokobori T, Oyama T, Nishiyama M, Kuwano H, Shirabe K. Relationship between LAT1 expression and resistance to chemotherapy in pancreatic ductal adenocarcinoma. Cancer Chemother Pharmacol. 2018; 81(1):141-53.

\section{Publisher's Note}

Springer Nature remains neutral with regard to jurisdictional claims in published maps and institutional affiliations.

Ready to submit your research? Choose BMC and benefit from:
- fast, convenient online submission
- thorough peer review by experienced researchers in your field
- rapid publication on acceptance
- support for research data, including large and complex data types
- gold Open Access which fosters wider collaboration and increased citations
- maximum visibility for your research: over 100M website views per year
At BMC, research is always in progress.
Learn more biomedcentral.com/submissions

